\title{
Rate and Modifiable Predictors of 30-Day Readmission in Patients with Acute Respiratory Distress Syndrome in the United States
}

Harshil Shah ${ }^{1}$, Uvesh Mansuri ${ }^{2}$, Sukrut Pagad ${ }^{3}$, Reshmi Adupa ${ }^{4}$, Jagmeet Singh ${ }^{5}$, Khin Tun ${ }^{6}$, Chail Shah 7,8 , Solomon Tuonuur ${ }^{9}$, Priyal J. Shah ${ }^{10}$, Mir Z. Ali Khan ${ }^{11}$, Gurjot S. Grewal ${ }^{12}$, Ruchir Goswami ${ }^{13}$, Shantanu Solanki ${ }^{14}$

1. Internal Medicine, Independent Researcher, Sayre, USA 2. Medicine, MedStar Union Memorial Hospital, Baltimore, USA 3. Internal Medicine, Larkin Community Hospital, Hialeah, USA 4. Internal Medicine, Garden City Hospital, Garden City, USA 5. Nephrology, Geisinger Commonwealth School of Medicine, Scranton, USA 6. Pediatrics, Independent Researcher, Yangon, MMR 7. Internal Medicine, Brooklyn Cancer Care, Brooklyn, USA 8. Internal Medicine, Mahatma Gandhi Medical College and Research Institute, Navi Mumbai, IND 9. Internal Medicine, University of New Haven, Meriden, USA 10. Internal Medicine, The Medical Center, Navicent Health, Macon, USA 11. Internal Medicine, Mercy Catholic Medical Center, Darby, USA 12. Medicine, Christian Medical College \& Hospital, Ludhiana, IND 13. Epidemiology and Public Health, Icahn School of Medicine at Mount Sinai, New York, USA 14. Hospital-Based Medicine, Geisinger Commonwealth School of Medicine, Scranton, USA

Corresponding author: Harshil Shah, harshilshah106@gmail.com

\section{Abstract}

\section{Background}

The 30-day readmission rates are being used as a quality measure by Centers for Medicare and Medicaid Services (CMS) for specific medical and surgical conditions. Acute respiratory distress syndrome (ARDS) is one of the important causes of morbidity and mortality in the United States (US). The characteristics and predictors of 30-day readmission in ARDS patients in the US are not widely known, which we have depicted in our study.

\section{Objective}

The aim of this study is to identify 30-day readmission rates, characteristics, and predictors of ARDS patients using the largest publicly available nationwide database.

\section{Methods}

We used the National Readmission Database from the year 2013 to extract the patients with ARDS by primary discharge diagnosis with ICD9-CM codes. All-cause unplanned 30-day readmission rates were calculated for patients admitted between January and November 2013. The independent predictors for unplanned 30-day readmission were identified by survey logistic regression.

Received 06/10/2020

Review began 06/18/2020 Review ended 06/23/2020 Published 06/30/2020

\section{() Copyright 2020}

Shah et al. This is an open access article distributed under the terms of the Creative Commons Attribution License CC-BY 4.0., which permits unrestricted use, distribution, and reproduction in any medium, provided the original author and source are credited.

\section{Results}

After excluding elective readmission, the all-cause unplanned 30-day readmission rate for ARDS patients was $18 \%$. Index admissions readmitted within 30-day had a significantly higher baseline burden of comorbidities with a Charlson Comorbidity Index (CCI) $\geqslant 1$ as compared to those who were not readmitted within 30 days. In multivariate regression analysis, several predictors associated with 30-day readmission were self-pay/no charge/other (OR 1.19, 95\%CI: 1.02-1.38; $p=0.02$ ), higher-income class (OR 0.86, 95\%CI:0.79-0.99; $p=0.03$ ), private insurance (OR 0.81, 95\%CI:0.67-0.94; $p=0.01$ ), and teaching metropolitan hospital (OR 0.72, 95\%CI:0.61-0.94; $p=0.01$ ).

\section{Conclusion}

The unplanned 30-day readmission rates are higher in ARDS patients in the US. Several modifiable factors such as insurance, socioeconomic status, and hospital type are associated with 30-day readmission among ARDS patients.

Categories: Internal Medicine, Pulmonology, Epidemiology/Public Health

Keywords: acute respiratory distress syndrome, readmission, predictors

\section{Introduction}

Acute respiratory distress syndrome (ARDS) is a syndrome with various etiologies characterized by lung inflammation, increased permeability, pulmonary edema, hypoxemia, and decreased lung compliance [1]. Clinical hallmarks of ARDS are hypoxemia and bilateral radiographic opacities, while the pathological 
hallmark is diffuse alveolar damage [1]. The clinical features of ARDS usually appear within 6 to 72 hours of an inciting event and worsen rapidly [1]. In a cohort study by Cheung et al., researchers attempted to determine the long-term outcomes of survivors of ARDS and it was determined that ARDS survivors continue to have a functional impairment and compromised health-related quality of life 2 years after discharge from the ICU [2]. Furthermore, patients who require mechanical ventilation in ARDS have a higher severity of illness and a higher number of comorbidities than their non-mechanically ventilated counterparts [3]. These patients also have higher lengths of stay, thus driving up total costs regardless of stable daily costs after the first two days [4-5]. Given the high cost of ARDS management and its lifethreatening implications, it is of utmost importance to explore the causes and predictors of 30-day readmission in ARDS patients. These are currently unknown to the US population. The objective of this study was to identify the 30-day readmission rates and modifiable predictors of 30-day readmission in ARDS patients using the largest publicly available nationwide database from the US.

\section{Materials And Methods}

\section{Data source}

Our retrospective observational study was derived from the subset of the Healthcare Cost and Utilization Project (HCUP) sponsored by the Agency for Healthcare Research and Quality (AHRQ) [6]. The National Readmission Database (NRD) is one of the largest publicly available all-payer inpatient care databases in the United States, including data on approximately 14 million discharges in the year 2013, estimating roughly 36 million weighted discharges from 21 states with reliable, verified linkage numbers [6-7]. NRD represents $49 \%$ of total US hospitalizations [7]. Patients were tracked using variable "NRD_Visitlink" used to verify patient linkage number for linking hospital visits for the same patient across hospitals and time between two admissions was calculated by subtracting time variable “NRD_DaysToEvent.” Time to readmission was calculated by subtracting the length of stay (LOS) of primary admissions to time between 2 admissions [8-9]. National estimates are produced using sampling weights provided by the NRD. The details regarding the NRD data are available online [7-8].

\section{Study population and design}

All variables were identified using the International Classification of Diseases, Ninth Revision, Clinical Modification (ICD-9-CM) Volume 3 diagnosis codes. We queried the NRD database using the presence of ICD-9-CM diagnosis code of 518.82 for ARDS in primary or secondary diagnostic fields which only includes ARDS related to non-trauma or surgical causes. We identified 24,307 ARDS patients (weighted N=53,555) after excluding patients with missing data for age or gender. We also excluded procedures done in the month of December, as we did not have follow-up data for the same, data points with LOS of 0 days were also excluded. Figure 1 shows the sequential derivation of the study cohort. Patients who were readmitted to the hospital within 30 days within the same calendar year were further evaluated.

\section{Outcome variables}

The primary outcome was 30-day readmission rate. Secondary outcomes included predictors of readmission in ARDS patients. NRD variables were used to identify patients' demographic characteristics including age, gender, hospital characteristics such as bed size and teaching status, and other patient-specific characteristics including median household income category for patient's zip code, primary payer, admission type, admission day, and discharge disposition [8]. We identified various co-morbid conditions as it is provided by AHRQ co-morbidity ( $\left.\mathrm{cm}_{-}\right)$measures with the database. Additionally, Deyo's modification of the Charlson comorbidity index (CCI) was used to define the severity of co-morbid conditions [10-12]. Deyo modification of Charlson co-morbidity index (CCI), which contains 17 co-morbid conditions was used as a measure of co-morbidity burden [10]. With score ranges from zero to 33, a higher score means a greater burden of co-morbid diseases [10].

\section{Statistical analysis}

We utilized Statistical Analysis Software (SAS) 9.4 (SAS Institute, Cary, NC, USA) for all analyses. Survey procedures were implemented to adjust for stratified cluster design of NRD with DOMAIN, STRATA, CLUSTER, and WEIGHT statements [13]. We used the $X^{2}$ test for categorical variables, t-test for normally distributed continuous variables, and the Wilcoxon rank-sum test for non-normally distributed continuous variables to compare the baseline characteristics of the study population. P-values less than 0.05 were considered significant. The independent predictors of unplanned 30-day readmission were identified by multivariate logistic regression adjusting for stratified cluster design of NRD. Multivariate models for readmission included hospital-level variables such as age, gender, admission type (elective vs. non-elective), median household income (higher income quartile vs. lowest income quartile), the primary payer (private insurance vs. self-pay vs. Medicaid/Medicare). The multivariate model for readmission was run only on patients who survived index admission. All interactions were thoroughly tested. Few observations and response variables were deleted due to missing or invalid values for its explanatory, frequency, weight, strata, or cluster variables. 


\section{Cureus}

\section{Results}

\section{Readmission rate}

There were 24,307 (weighted $\mathrm{N}=53,555$ ) index admissions for ARDS in the US during the study period out of which, 4,470 (weighted $\mathrm{N}=9,807$ ) patients were readmitted within 30 days, which inferred the readmission rate at $18 \%$ (Figure 1 ).

HCUP's National Readmission Database (NRD) of 2013 was used

\section{2}

Patients with Acute respiratory distress syndrome (ARDS) were identified by ICD-9-CM Codes: 518.82 in primary and secondary discharge diagnosis filed $(\mathrm{N}=33,336$, Weighted $=77,932)$

\{.

Patients with admissions less than age 18 years were removed $(N=29,895$, Weighted $=66,118)$

\2

Patients with admissions in month of December were removed $(\mathrm{N}=27,099$, Weighted $=59,857)$

Y.

Patients with missing data on Age, Gender and Died were removed $(N=24,313$, Weighted $=53,565)$

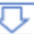

Index admission is defined by patients who have not missed LOS, time variable 'NRD DaysToEvent', admitted between the months of $01-11$, discharged alive, ICD 9 CM code for ARDS. \.

Total number of Index Admissions $\mathrm{N}=24,307$, Weighted N=53,555

$$
\text { Y }
$$

All index admissions were followed over 30 days post discharge using patient linkage variable "NRD_VisitLink" and time variable "NRD DaysToEvent" ₹.

Total no. of 30 days Readmissions $\mathrm{N}=4,470$, Weighted $\mathrm{N}=9,807$

Readmission rate is $18 \%$ (readmitted $\mathrm{N}=4470 /$ Index admissions $\mathrm{N}=24,307$ )

FIGURE 1: Sequential derivation of study population

Baseline characteristics of index admissions stratified by presence of

\section{0-day readmission}

Index admissions who were readmitted within 30-day had a significantly higher baseline burden of comorbidities with a CCI $\geqslant 1$ ( $87 \%$ vs. $81 \% ; p<0.001)$ as compared to those who did had 30-day readmission. Similarly, comorbidities like hypertension (63\% vs $60 \%$; $p<0.001$ ), electrolyte imbalance ( $50 \%$ vs $43 \%$; $p<$ 0.001 ), renal failure ( $28 \%$ vs. $20 \%$; $p<0.001$ ), and diabetes mellitus ( $27 \%$ vs $24 \%$; $p<0.001$ ) were higher among those index admissions who had 30-day readmission. The index admissions that had readmission were more likely to be from large bed size hospital (67\% vs. $64 \%$; $p<0.001$ ), were admitted non-electively (93\% vs. $90 \%$; p <0.001) and had Medicare as an insurance (67\% vs. 63\%; $p<0.001)$. Additionally, an index admission with readmission had higher proportion of discharge disposition to facility (32\% vs. $26 \% ; p<$ 0.001 ) and LOS $\geqslant 7$ days ( $48 \%$ vs. $40 \% ; p<0.001$ ) than those without readmits. Other characteristics, including age, gender, and hospital teaching status were approximately evenly distributed among both groups. Another detailed distribution of patient and hospital-level characteristics has been depicted in Table 1.

\begin{tabular}{|c|c|c|c|c|}
\hline & Index Admission & No Readmission & Readmission & $p$ value \\
\hline Total population (unweighted) & 24,307 & 19,837 & 4,470 & \\
\hline Total population (weighted) & 53,555 & 43,748 & 9,807 & \\
\hline \multicolumn{5}{|l|}{ Patient level variables } \\
\hline Age (\%) & & & & 0.08 \\
\hline$\geq 85$ & 14 & 14 & 13 & \\
\hline 65-84 & 29 & 29 & 30 & \\
\hline
\end{tabular}




\section{Cureus}

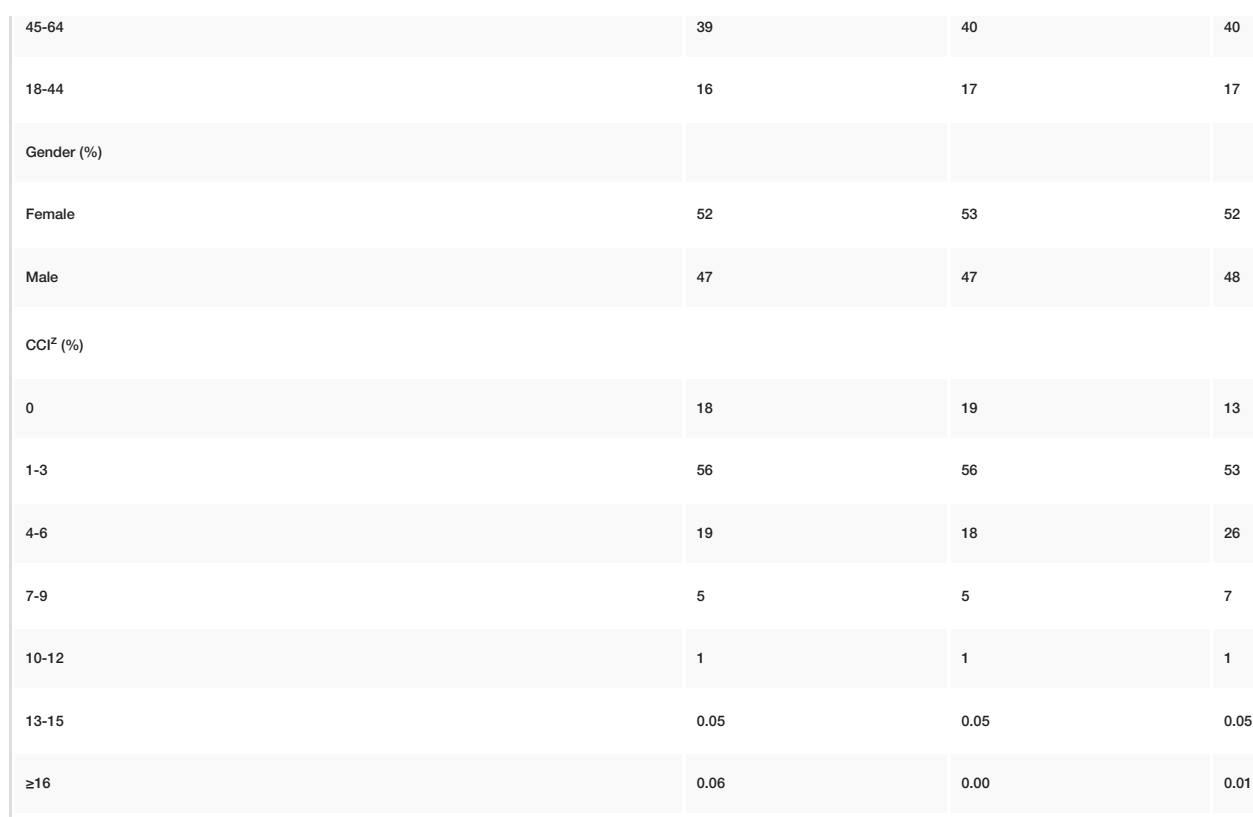

Length of stay $(\%)$

1-3

4-6

7-9

10-12

13-15

$\geq 16$

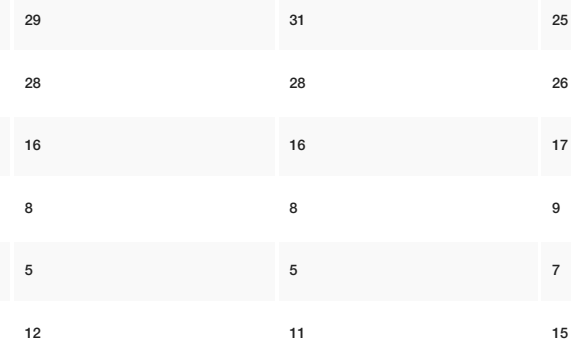

Comorbidities (\%) *

Drug abuse

Hypertension

Hypothyroidism

Liver disease

Fluid and electrolyte disorders

Metastatic cancer

Neurological disorders

Obesity

Peripheral vascular disorders

Psychoses

Pulmonary circulation disorders

Renal Failure

Valvular disease

Alcohol abuse

Rheumatoid arthritis/ collagen vascular diseases

Chronic blood loss anemia

Congestive heart failure

Chronic pulmonary disease

Depression

0.01
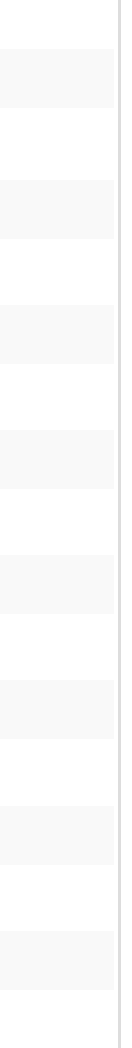

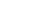

.58




\section{Cureus}

\begin{tabular}{|c|c|c|c|c|}
\hline Diabetes, uncomplicated & 25 & 24 & 27 & 0.002 \\
\hline Diabetes with chronic complications & 7 & 7 & 10 & \\
\hline Median household income $(\%)^{x}$ & & & & 0.1 \\
\hline $76-100^{\text {th }}$ & 27 & 26 & 28 & \\
\hline $51-75^{\text {th }}$ & 26 & 26 & 24 & \\
\hline $26-50^{\text {th }}$ & 25 & 25 & 25 & \\
\hline $0-25^{\text {th }}$ & 21 & 22 & 21 & \\
\hline \multicolumn{5}{|l|}{ Primary Payer (\%) } \\
\hline Self-pay/no charge/other & 63 & 63 & 67 & \\
\hline Private including $\mathrm{HMO}^{\#}$ & 11 & 10 & 13 & \\
\hline Medicaid & 18 & 19 & 15 & \\
\hline Medicare & 8 & 8 & 5 & \\
\hline Hospital bed size $(\%)^{r}$ & & & & 0.02 \\
\hline Large & 11 & 11 & 10 & \\
\hline Medium & 24 & 25 & 23 & \\
\hline Small & 65 & 64 & 67 & \\
\hline Hospital teaching status $(\%)$ & & & & 0.05 \\
\hline Non-metropolitan hospital & 32 & 32 & 32 & \\
\hline Metropolitan teaching & 58 & 58 & 59 & \\
\hline Metropolitan non-teaching & 10 & 10 & 9 & \\
\hline \multicolumn{5}{|l|}{ Admission type (\%) } \\
\hline Elective & 91 & 90 & 93 & \\
\hline Non elective & 9 & 10 & 7 & \\
\hline \multicolumn{5}{|l|}{ Disposition (\%) } \\
\hline Against medical advice & 50 & 52 & 42 & \\
\hline Facilities & 22 & 21 & 24 & \\
\hline Home health care & 27 & 26 & 32 & \\
\hline Home & 1 & 1 & 2 & \\
\hline
\end{tabular}

\section{TABLE 1: Baseline characteristics of index admissions stratified by presence of 30 -day}

\section{readmission}

z - Charlson/Deyo co-morbidity index (CCl) was calculated as per Deyo classification.

* Variables are AHRQ co-morbidity $\left(\mathrm{cm} \_\right.$) measures.

$\mathrm{x}$ - Represents a quartile classification of the estimated median household income of residents in the patient's ZIP Code, derived from ZIP Codedemographic data obtained from Claritas. The quartiles are identified by values of 1 to 4 , indicating the poorest to wealthiest populations. Because these estimates are updated annually, the value ranges vary by year. http://www.hcupus.ahrq.gov/db/vars/zipinc_qrtl/nisnote.jsp.

\# -HMO: Health Maintenance Organization.

\{- The bed size cutoff points divided into small, medium, and large have been done so that approximately one-thirdof the hospitals in a given region, location, and teaching status combination would fall within each bed size category. State and County QuickFacts. Washington, DC: US Census Bureau; 2012. 


\section{Cureus}

\section{Predictors of 30-day readmission}

From our analysis, several predictors associated with increased 30-day readmission were higher CCI (OR for $\geqslant 3$ 1.147, 95\%CI: 1.065-1.236; $p<0.001$ ), self-pay/no charge/other (OR 1.19, 95\%CI: 1.02-1.38; $p=0.02$ ), discharge disposition against medical advice (OR 1.25, 95\%CI:1.12-1.39; $p<0.001$ ), and discharge disposition to facility (OR 1.28, 95\%CI 1.15-1.43; $p<0.001$ ). Meanwhile, we also found several predictors associated with reduced odds for 30-day readmission: higher income class (OR 0.86, 95\%CI:0.79-0.99; $p=$ 0.03 ), private insurance (OR 0.81, 95\% CI:0.67-0.94; $p=0.01$ ), and teaching metropolitan hospital (OR 0.72, 95\% CI:0.61-0.94; $p=0.01)$. On the other hand, several characteristics were not found to be associated with increased or decreased readmission (Table 2 ).

\begin{tabular}{|c|c|c|c|}
\hline Predictors & Odds Ratio & $95 \% \mathrm{Cl}$ & $p$ value \\
\hline \multicolumn{4}{|l|}{ Age } \\
\hline$\geq 85$ & 0.97 & $0.81-1.15$ & 0.67 \\
\hline $65-84$ & 0.75 & $0.61-0.92$ & 0.001 \\
\hline 45-64 & 0.69 & $0.55-0.88$ & 0.0002 \\
\hline 18-44 & Ref & & \\
\hline \multicolumn{4}{|l|}{ Gender } \\
\hline Female & 0.96 & 0.88-1.05 & 0.43 \\
\hline Male & Ref & & \\
\hline \multicolumn{4}{|l|}{$\mathrm{CCl}^{2}$} \\
\hline$\geq 16$ & 1.29 & $1.14-1.46$ & \\
\hline 13-15 & 1.39 & $1.14-1.70$ & \\
\hline 10-12 & 1.24 & $0.87-1.76$ & 0.23 \\
\hline 7-9 & 0.61 & $0.08-4.73$ & 0.64 \\
\hline $4-6$ & 1.98 & $0.14-28.82$ & 0.62 \\
\hline $0-3$ & Ref & & \\
\hline \multicolumn{4}{|l|}{ Length of stay } \\
\hline$\geq 16$ & 1.16 & $1.03-1.31$ & 0.01 \\
\hline 13-15 & 1.26 & $1.11-1.44$ & 0.0003 \\
\hline 10-12 & 1.27 & 1.09-1.45 & 0.003 \\
\hline 7-9 & 1.51 & $1.26-1.78$ & \\
\hline $4-6$ & 1.39 & $1.19-1.62$ & \\
\hline $1-3$ & Ref & & \\
\hline \multicolumn{4}{|l|}{ Comorbidities* $^{*}$} \\
\hline Drug abuse & 1.23 & $0.96-1.57$ & 0.11 \\
\hline Hypertension & 1.04 & $0.94-1.14$ & 0.49 \\
\hline Hypothyroidism & 1.14 & $1.02-1.27$ & 0.02 \\
\hline Liver disease & 1.19 & $0.99-1.42$ & 0.06 \\
\hline Fluid and electrolyte disorders & 1.09 & $1.00-1.19$ & 0.16 \\
\hline Metastatic cancer & 1.09 & $0.85-1.38$ & 0.3 \\
\hline Neurological disorders & 1.03 & $0.88-1.19$ & 0.49 \\
\hline Obesity & 1.01 & 0.89-1.14 & 0.73 \\
\hline
\end{tabular}




\section{Cureus}

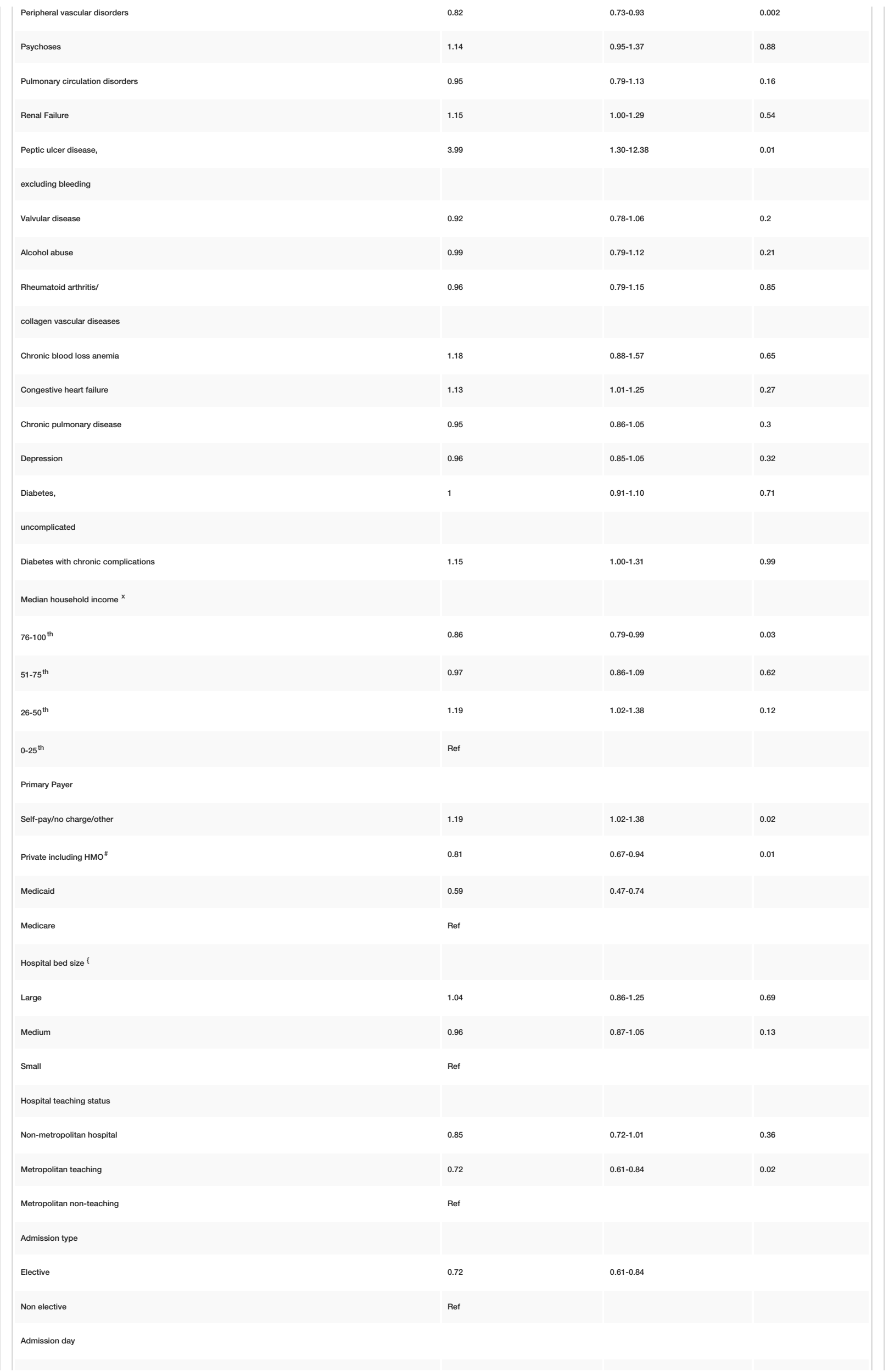




\section{Cureus}

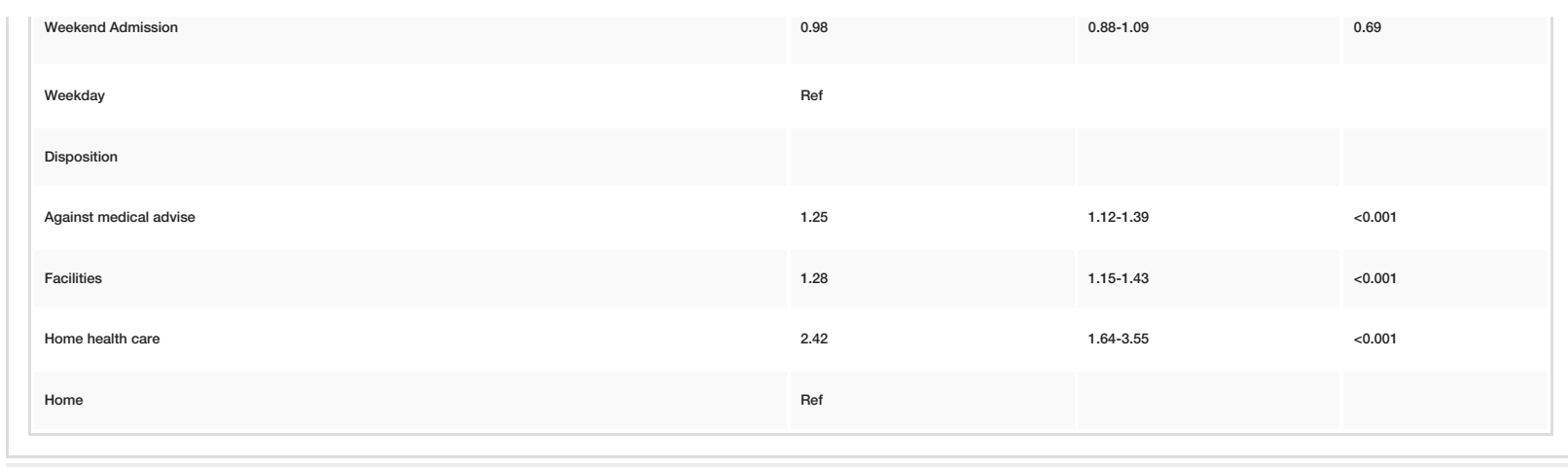

\section{TABLE 2: Multivariate analysis in 30-day readmission in ARDS patients}

z - Charlson/Deyo co-morbidity index (CCl) was calculated as per Deyo classification.

*Variables are AHRQ co-morbidity (cm_) measures.

$\mathrm{x}$ - Represents a quartile classification of the estimated median household income of residents in the patient's ZIP Code, derived from ZIP Codedemographic data obtained from Claritas. The quartiles are identified by values of 1 to 4 , indicating the poorest to wealthiest populations. Because these estimates are updated annually, the value ranges vary by year. http://www.hcupus.ahrq.gov/db/vars/zipinc qrtl/nisnote.jsp.

\# -HMO: Health Maintenance Organization. \{- The bed size cutoff points divided into small, medium, and large have been done so that approximately one-third of the hospitals in a given region, location, and teaching status combination would fall within each bed size category. State and County QuickFacts. Washington, DC: US Census Bureau; 2012.

ARDS, acute respiratory distress syndrome

\section{Discussion}

We report contemporary data from an NRD on readmission of patients with ARDS. Our study reports an allcause unplanned 30-day readmission rate of $18 \%$ in ARDS patients. A Canadian study on long-term outcomes of ARDS has an estimated 39\% readmission in the first 2 years after the discharge [2]. This study was from four academic tertiary care ICUs from a city [2]. However, our study is based on a large administrative database, which can be weighted to produce national estimates. Our study also highlights that ARDS patients who got readmitted in 30 days had a higher burden of co-morbidities especially hypertension, electrolyte disorders, and diabetes. Pan et al. found that higher CCI is associated with the increased risk of admission which we have also repopulated in our study, CCI $\geqslant 3$ (OR 1.147, 95\%CI: 1.0651.236; $\mathrm{p}<0.001$ ) [14]. Furthermore, we found several other factors associated with increased 30-day readmission. We observed that patients who leave against medical advice showed a higher risk for unplanned 30-day readmission for ARDS. Furthermore, discharge to the facility was also a significant predictor of readmission in our study, which was also shown by Pan et al. [14]. This could be because the patients being discharged to facilities are likely sicker with a higher number of co-morbidities or newly acquired morbidity or system failure due to the effect of ARDS [15]. Further, we observed that self-pay/No charge/other had higher odds of unplanned 30-day readmission, and patients with the higher socioeconomic income class have the reduced odds of 30-day readmission, which signifies that socioeconomic factors might have played a role in leading to readmissions in the ARDS population. Meanwhile, Ferro EG et al. showed the percentage of readmissions was lower among the patients with private insurance in all-cause index hospitalizations [16]. Interestingly, we also observed a similar phenomenon in ARDS index hospitalizations. It is known that Medicaid patients have greater difficulty obtaining appointments in comparison to privately insured patients [17]. We postulate that privately insured patients have a better follow-up compared to others and hence, they had lower readmission rates in our study. We also found reduced odds of readmission in the patients at teaching metropolitan hospitals, which can be attributed to the advanced medical care and better implementation of the quality improvement programs in the academic settings. Khaksari BJ et al. showed no association between weekend admission and 30-day readmission, which was seen in our study as well [18].

Our study has several limitations. First, our study is a retrospective study which makes it subjectable to selection bias. Secondly, the selection of samples relies on the sensitivity and specificity of ICD-9-CM codes which could have confounded our results. Also, the NRD database does not contain information on the long-term follow-up of the patients. Our study also lacks data on ARDS patients discharged to home hospice who are not at risk of being readmitted, which might result in an underestimation of the readmission rate. Despite its limitations, our study has several strengths. Our study is the first nationwide study looking at readmission rates and predictors of ARDS. Our sample size most closely represents the standardized U.S. population. Targets for future interventions should be focused on better risk stratification and controlling the predictors that aggravate the readmission rates of ARDS, which we were able to identify successfully. 


\section{Conclusions}

The unplanned 30-day readmission rates are higher for ARDS patients in the US. There are several modifiable factors such as type of insurance, socioeconomic status, and hospital type associated with 30-day readmission among ARDS patients. Further studies are needed to identify preventable readmissions, aim to modify the predictors, and develop a strategic approach to reduce the burden of readmissions in patients admitted with ARDS.

\section{Additional Information \\ Disclosures}

Human subjects: All authors have confirmed that this study did not involve human participants or tissue. Animal subjects: All authors have confirmed that this study did not involve animal subjects or tissue. Conflicts of interest: In compliance with the ICMJE uniform disclosure form, all authors declare the following: Payment/services info: All authors have declared that no financial support was received from any organization for the submitted work. Financial relationships: All authors have declared that they have no financial relationships at present or within the previous three years with any organizations that might have an interest in the submitted work. Other relationships: All authors have declared that there are no other relationships or activities that could appear to have influenced the submitted work.

\section{Acknowledgements}

Author Contributions: H.S. and U.M. gave equal contributions.

\section{References}

1. Warrell DA, Cox TM: Firth JD: Oxford Textbook of Medicine . 2010. 10.1093/med/9780199204854.001.1

2. Cheung AM, Tansey CM, Tomlinson G, et al.: Two-year outcomes, health care use, and costs of survivors of acute respiratory distress syndrome. Am J Respir Crit Care Med. 2006, 174:538-544. 10.1164/rccm.200505$6930 \mathrm{C}$

3. Wunsch H: Three-year outcomes for medicare beneficiaries who survive intensive care. JAMA. 2010, 303:849-56. 10.1001/jama.2010.216

4. Kahn JM, Rubenfeld GD, Rohrbach J, Fuchs BD: Cost savings attributable to reductions in intensive care unit length of stay for mechanically ventilated patients. Medical Care. 2008, 46:1226-1233. 10.1097/MLR.0b013e31817d9342

5. Shwartz M, Iezzoni LI, Moskowitz MA, Ash AS, Sawitz E: The importance of comorbidities in explaining differences in patient costs. Medical Care. 1996, 34:767-782. 10.1097/00005650-199608000-00005

6. Overview of HCUP. (2020). Accessed: May 7, 2020: https://www.ahrq.gov/data/hcup/index.html.

7. Overview of the Nationwide Readmissions Database (NRD) . (2020). Accessed: May 6, 2020: https://www.hcup-us.ahrq.gov/nrdoverview.jsp.

8. NRD Description of Data Elements . (2020). Accessed: May 7, 2020: https://www.hcupus.ahrq.gov/db/nation/nrd/nrddde.jsp.

9. Bhatt P, Dave M, Amponsah JK, et al.: Etiologies, trends, and predictors of 30-day pediatric readmissions after hospitalizations for diabetic ketoacidosis in the United States [published online ahead of print, 2020 May 29]. Pediatr Diabetes. 2020, 10.1111/pedi.13059. 10.1111/pedi.13059

10. Charlson ME: Why predictive indexes perform less well in validation studies . Arch Intern Med. 1987, 147:2155-2161. 10.1001/archinte.1987.00370120091016

11. Charlson ME, Pompei P, Ales KL, MacKenzie CR: A new method of classifying prognostic comorbidity in longitudinal studies: development and validation. J Chronic Dis. 1987, 40:373-383. 10.1016/00219681(87)90171-8

12. Romano PS, Geppert JJ, Davies S, Miller MR, Elixhauser A, McDonald KM: A national profile of patient safety in U.S. hospitals. Health Affairs. 2003, 22:154-166. 10.1377/hlthaff.22.2.154

13. HCUP Statistical Briefs - Readmissions and Revisits. (2020). Accessed: May 6, 2020: https://www.hcupus.ahrq.gov/reports/statbriefs/sb_readmission.jsp.

14. Pan D, Salguero B, Chen L, Vazquez de Lara F: Analysis of readmission outcomes and morbidity in survivors of ARDS. Chest. 2019, 156:10.1016/j.chest.2019.08.459

15. Herridge, Margaret S: Fifty years of research in ARDS. Long-term follow-up after acute respiratory distress syndrome. Insights for managing medical complexity after critical illness. Am J Respir Crit Care Med. 2017, 196:1380-84. https://doi.org/10.1164/rccm.201704-0815ED

16. Ferro EG, Secemsky EA, Wadhera RK, et al.: Patient readmission rates for all insurance types after implementation of the hospital readmissions reduction program. Health Affairs. 2019, 38:585-593. 10.1377/hlthaff.2018.05412

17. Hsiang WR, Lukasiewicz A, Gentry M, et al.: Medicaid patients have greater difficulty scheduling health care appointments compared with private insurance patients: a meta-analysis. INQUIRY: The Journal of Health Care Organization, Provision, and Financing. 2019, 56. 10.1177/0046958019838118

18. Khaksari Bijan J, Kulick Erin R, Elkind Mitchell SV, Boehme Amelia K: “Weekend Effect” on 30-day readmissions among stroke survivors: an analysis of the National Readmission Database. Cerebrovasc Dis Extra. 2019, 9:66-71. 10.1159/000500611 